\title{
PACKET SCHEDULING AGAINST STEPPING-STONE ATTACKS WITH CHAFF
}

\author{
Ting He, Parvathinathan Venkitasubramaniam, and Lang Tong \\ School of Electrical and Computer Engineering \\ Cornell University \\ Email: $\{$ th255, pv45, 1t35\}@cornell.edu
}

\begin{abstract}
We consider scheduling packet transmissions in a network so that the efficiency of stepping-stone attacks can be severely restrained with the help of stepping-stone monitors. We allow the attacker to encrypt and pad the packets, perturb the timing of packets, and insert chaff packets, but the timing perturbation is subject to a maximum delay constraint. We show that if we randomize packet transmissions, then the attacker has to insert a large amount of chaff to evade detection completely. In particular, if all transmissions are scheduled as Poisson processes, then the fraction of attacking packets in the attacker's traffic decreases exponentially with the length of the intrusion path.
\end{abstract} ing.

Index Terms - Stepping-stone attack, Network defense, Schedul-

\section{INTRODUCTION}

Stepping-stone attacks are indirect network attacks in which attacking commands are relayed through compromised hosts called "stepping stones" [1]. Since each stepping stone host only sees its immediate predecessor and the victim only sees the last host, it is difficult to find the origin of such attacks. The key to defending against stepping-stone attacks is to find the intrusion path.

Although numerous detection schemes have been developed to detect stepping-stone connections, a sophisticated attacker can modify his traffic to evade detection. In particular, he can encrypt and pad the packets so that no information is revealed by the bit patterns or the lengths of packets; he can also perturb the timing of packets by adding random delay or packet reshuffling. Furthermore, the attacker can repacketize the commands, or mix attacking traffic with other traffic or dummy traffic called "chaff". The insertion of chaff makes the detection of stepping-stone traffic especially challenging. We refer to the traffic of attacking packets as attacking traffic, and the mixture of attacking traffic

\footnotetext{
${ }^{\dagger}$ This work is supported in part by TRUST (The Team for Research in Ubiquitous Secure Technology), which receives support from the National Science Foundation (NSF award number CCF-0424422) and the following organizations: Cisco, ESCHER, HP, IBM, Intel, Microsoft, ORNL, Qualcomm, Pirelli, Sun and Symantec, and the U. S. Army Research Laboratory under the Collaborative Technology Alliance Program, Cooperative Agreement DAAD19-01-2-0011. The U. S. Government is authorized to reproduce and distribute reprints for Government purposes notwithstanding any copyright notation thereon.
}

and chaff stepping-stone traffic.

\subsection{Related Work}

Staniford and Heberlein [1] were the first to consider the problem of detecting stepping-stone connections. Early techniques are based on the content of the traffic; see, e.g., [1,2]. These techniques, however, are not applicable to detecting encrypted connections. An alternative is to exploit timing characteristics of the traffic; examples include [3-5]. The drawback of these schemes is that they are vulnerable to active timing perturbation by the attacker.

There are a few results on detecting encrypted, timing perturbed stepping-stone connections; see [6-9]. The key assumption of these methods is that the attacker is able to perform a packet-conserving transformation subject to certain constraints.

Packet conservation is too restrictive without considering the presence of chaff. We are only aware of a few results dealing with the attacker's insertion of chaff packets. Peng et al. in [10] proposed an active detection scheme which combines watermarking with packet matching to detect stepping-stone traffic with chaff. They assumed that packets have bounded delays, and chaff only appears in the outgoing stream. Their scheme injects watermarks in the incoming stream, and finds a subsequence in the outgoing stream, whose watermark is closest to the injected one. Such a scheme, however, requires the active manipulation of traffic. Donoho et al. [6] pointed out that in principle it is possible to correlate stepping-stone traffic even if both (bounded) delay and independent chaff are introduced during the relay. Blum et al. [8] proposed an algorithm called "DETECT-ATTACKS-CHAFF" (DAC) to detect stepping-stone traffic with limited chaff when attacking traffic has bounded delay and bounded peak rate. Algorithm DAC monitors the difference in the number of packets in the incoming and the outgoing streams, and makes detection if the difference exceeds a certain threshold. The algorithm achieves robustness against a limited number of chaff packets by choosing a threshold larger than necessary. The drawback is that the increase of threshold causes increased false alarm probability, and the attacker can still evade de- 
tection by adding a fixed number of chaff packets. In a recent paper [11], Zhang et al. proposed packet matching schemes to detect stepping-stone traffic with bounded delay perturbation and chaff. They proposed to match every arrival with the first departure subject to causality and the delay constraint. They proved that this strategy has exponentially decaying false alarm probability for independent Poisson streams. Their schemes can detect stepping-stone traffic if chaff is only inserted in the departing stream. If chaff can be inserted in the incoming stream, however, one chaff packet suffices to evade their schemes.

\subsection{Summary of Results and Organization}

In this paper, we show that there are fundamental limits to stepping-stone attacks even if the attacker can encrypt and pad the packets, perturb the timing, and mix attacking packets with chaff. Based on these limits, we propose a randomized packet scheduling strategy to defend against steppingstone attacks more efficiently.

We consider encrypted stepping-stone attacks with bounded delay perturbation and chaff. We first analyze the fundamental limits on how fast the attacker can send attacking traffic without being detected by any stepping-stone detector. We propose optimal strategies to schedule the transmission of attacking packets for given realizations of arrival processes while inserting the minimum number of chaff packets. Then the fundamental limits on the rate of the attacking traffic are obtained by characterizing the performance of the proposed chaff-inserting algorithms. We show that although the attacker does not lose much rate in one-hop stepping-stone attacks, the rate of attacking traffic decreases exponentially as the number of hops increases. This result suggests that in detecting stepping-stone traffic, we should jointly consider streams at multiple locations rather than doing local detection separately.

We then compare the achievable rates of the attacking traffic under randomized packet scheduling versus deterministic scheduling. The comparison suggests that randomized packet transmissions can make the network much more robust to stepping-stone attacks.

The rest of the paper is organized as follows. Section 2 defines the problem. Section 3 gives a limit on the rate of attacking traffic passing through a single stepping-stone host. In Section 4, the result is generalized to the case of multiple stepping-stone hosts. Section 5 presents how randomized packet scheduling can facilitate stepping-stone detection. Finally, Section 6 concludes the paper with comments on its limitation.

\section{PROBLEM STATEMENT}

Let the packet arrivals on stream $i$ be represented by a point process

$$
S_{i}=\left(s_{1}^{(i)}, s_{2}^{(i)}, s_{3}^{(i)}, \ldots\right), \quad i=1,2, \ldots,
$$

where $s_{k}^{(i)}$ is the $k$ th arrival epoch of stream $i$. Let $\mathcal{T}_{i}=$ $\left\{s_{1}^{(i)}, s_{2}^{(i)}, \ldots\right\}$ be the set of the elements in $S_{i}$. Let $S_{1}$ be an incoming stream of the first host, and $S_{i+1}(i=1, \ldots, n)$ be a outgoing stream at the $i$ th host. The outgoing stream at the $i$ th host is different from the incoming stream at the $i+1$ th host due to perturbations from clock skews and propagation delay. We assume that these perturbations are known.

Normally, $S_{i}$ 's are independent. If, however, $\left(S_{i}\right)_{i=1}^{n+1}$ is a sequence of stepping-stone streams on the same intrusion path, then they will satisfy certain relation as defined below.

Definition 1 A sequence of streams $\left(S_{1}, \ldots, S_{n+1}\right)$ is normal traffic if they are independent. It is attacking traffic if there exist bijections $g_{i}: \mathcal{T}_{i} \rightarrow \mathcal{T}_{i+1}(i=1, \ldots, n)$ such that $g_{i}(s)-s \geq 0$ for all $s \in \mathcal{T}_{i}$. Furthermore, if there exists a constant $\Delta<\infty$ such that the bijections satisfy $g_{i}(s)-s \leq \Delta$ for all $s \in \mathcal{T}_{i}(i=1, \ldots, n)$, then $\left(S_{1}, \ldots, S_{n+1}\right)$ is attacking traffic with bounded delay $\Delta$.

The bijection $g_{i}$ is a mapping between the arrival and the departure times of packets at the $i$ th host, allowing permutation of packets during the relay. The condition that $g_{i}$ is a bijection imposes a packet-conservation constraint, i.e., no packets are generated or dropped at the stepping stones. The condition $g_{i}(s)-s \geq 0$ is the causality constraint, which means that a packet cannot leave a host before it arrives. The condition $g_{i}(s)-s \leq \Delta$ means that packets can stay at a stepping-stone host for at most $\Delta$, where $\Delta$ is referred to as the maximum tolerable delay. The bounded delay constraint is usually imposed by physical constraints on the communication link or the need of the attacker.

If the attacker can insert chaff into his traffic, then the above constraints only apply to the fraction of the traffic made of attacking packets, as stated in the following definition.

Definition 2 A sequence of streams $\left(S_{1}, \ldots, S_{n+1}\right)$ is steppingstone traffic if it is the superposition of attacking traffic and a sequence of chaff streams $\left(C_{1}, \ldots, C_{n+1}\right)$. Steppingstone traffic with bounded delay is similarly defined as the superposition of chaff and attacking traffic with bounded delay.

Stream $C_{i}(i=1, \ldots, n+1)$ consists of dummy packets called chaff which do not need to arrive at the destination. 
Chaff packets can be generated or dropped at any stepping stones without affecting the attack. They are artificially inserted by the attacker to evade detection.

We consider the centralized detection of the following hypotheses:

$$
\begin{aligned}
& \mathcal{H}_{0}:\left(S_{1}, \ldots, S_{n+1}\right) \text { is normal traffic, } \\
& \mathcal{H}_{1}:\left(S_{1}, \ldots, S_{n+1}\right) \text { is stepping-stone traffic, }
\end{aligned}
$$

by observing $\left(s_{1}^{(i)}, s_{2}^{(i)}, \ldots\right)_{i=1}^{n+1}$. In this paper, we focus on the detection of stepping-stone traffic with bounded delay.

\section{FUNDAMENTAL LIMIT ON ONE-HOP STEPPING-STONE ATTACKS}

In this section, we consider the simple case when $n=1$, i.e., there is only one stepping-stone host on the intrusion path. With enough chaff packets, the attacker can make his traffic look identical to any processes he wants. The problem is that the transmission of chaff packets causes a waste of rate. To launch attacks efficiently, the attacker will have the motivation to reduce the amount of chaff as much as possible.

Blum et al. in [8] propose an optimal chaff-inserting algorithm called "BOUNDED-GREEDY-MATCH" (BGM) which can embed a pair of stepping-stone streams with bounded delay into arbitrary point processes while inserting the minimum amount of chaff packets. Given a pair of incoming and outgoing streams at a host, BGM matches arrivals with departures subject to the constraints of causality and bounded delay. In [12], we combine the insertion of chaff and the transmission of attacking packets into the algorithm in Table 1 . Then for each valid pair $\left(s_{m}^{(1)}, s_{n}^{(2)}\right)$, the attacker can schedule an attacking packet to arrive at $s_{m}^{(1)}$ and depart at $s_{n}^{(2)}$.

Algorithm BGM has a low complexity of $O\left(\left|S_{1}\right|+\left|S_{2}\right|\right)$ because it only needs to scan $\left(S_{1}, S_{2}\right)$ once and the amount of work in each iteration is constant. It is shown in [8] that among all the algorithms that embed attacking packets into point processes subject to the bounded delay constraint, BGM inserts the minimum number of chaff packets $^{1}$. In [12], we characterize the minimum amount of chaff to mimic independent Poisson processes in the following theorem.

Theorem 1 If $S_{1}$ and $S_{2}$ are independent Poisson processes of equal rate $\lambda$, then $B G M$ inserts $1 /(1+\lambda \Delta)$ fraction of chaff among all the packets in $S_{1} \cup S_{2}$

\footnotetext{
${ }^{1}$ The original proof in [8] is for independent binomial processes, but it holds for arbitrary processes.
}

Table 1: BOUNDED-GREEDY-MATCH (BGM).

$$
\begin{aligned}
& \text { BOUNDED-GREEDY-MATCH }\left(S_{1}, S_{2}, \Delta\right) \\
& m=n=1 \text {; } \\
& \text { while } m \leq\left|S_{1}\right| \text { and } n \leq\left|S_{2}\right| \\
& \text { if } s_{n}^{(2)}-s_{m}^{(1)}<0 \\
& s_{n}^{(2)}=\text { chaff; } n=n+1 \text {; } \\
& \text { else if } s_{n}^{(2)}-s_{m}^{(1)}>\Delta \\
& \quad s_{m}^{(1)}=\text { chaff; } m=m+1 \text {; } \\
& \text { else } \left.{ }^{(1)}, s_{n}^{(2)}\right)=\text { a valid pair; } \\
& \quad m=m+1 ; n=n+1 ; \\
& \text { end } \\
& \text { end } \\
& \text { end }
\end{aligned}
$$

Remark: The theorem implies that the attacker can send attacking traffic at rate $\lambda^{2} \Delta /(1+\lambda \Delta)$, while keeping his traffic identical to independent Poisson processes of rate $\lambda$ by inserting chaff packets. For large $\lambda$, the attacker can send attacking traffic at rather high rate without possibly being detected by any activity-based detector.

\section{FUNDAMENTAL LIMIT ON MULTI-HOP STEPPING-STONE ATTACKS}

The result in Section 3 is pessimistic in that it seems possible that the detector has no way to detect encrypted one-hop stepping-stone attacks even if the attacker only transmits a small amount of chaff; it shows the weakness of detecting stepping-stone attacks on a local scale. If, however, the stepping-stone attack involves multiple hops, and there is a central detector which makes decisions based on the incoming and outgoing traffic at each hop, then the capability of the attacker to evade detection will be severely limited. We proceed by introducing a few definitions related to multihop stepping-stone attacks.

Definition $3 A$ relay path through a sequence of streams $\left(S_{1}, \ldots, S_{n+1}\right)$ is a sequence of epochs from each of the streams $\left(t_{i} \in S_{i}\right)_{i=1}^{n+1}$. A relay path $\left(t_{1}, \ldots, t_{n+1}\right)$ is valid for delay bound $\Delta$ if $t_{i+1}-t_{i} \in[0, \Delta]$ for all $i=1, \ldots, n$. $A$ set of relay paths is feasible if all the relay paths in it are disjoint and valid. A feasible set of relay paths is orderpreserving if any two paths in it $\left(t_{i}\right)_{i=1}^{n+1}$ and $\left(t_{i}^{\prime}\right)_{i=1}^{n+1}$ satisfy either $t_{i} \leq t_{i}^{\prime}$ for all $i$ or $t_{i} \geq t_{i}^{\prime}$ for all $i$.

A valid relay path represents a sequence of timestamps at which an attacking packet is emitted from each of the 
stepping-stone hosts. To schedule the transmission of attacking packets, the attacker must find a feasible set of relay paths, and schedule the transmission of each attacking packet according to a different relay path. The requirement that paths in a feasible set are disjoint is because we do not allow the combining of multiple packets into a single relay packet. If a set of relay paths is order-preserving, then there will be no intersection between the paths, which greatly reduces the complexity in searching for a desired set of relay paths.

Proposition 1 Among all the feasible sets of relay paths with the largest cardinality, there always exists a set which is order-preserving.

Remark: By Proposition 1, we only need to search among order-preserving sets to find a largest feasible set of relay paths.

Proof: The proof is by direct observation. As illustrated in Fig. 1, suppose $\left(s_{1}^{(1)}, s_{2}^{(2)}, s_{1}^{(3)}\right)$ and $\left(s_{2}^{(1)}, s_{1}^{(2)}, s_{2}^{(3)}\right)$ are valid relay paths. By switching the intersected part, we obtain two order-preserving paths $\left(s_{1}^{(1)}, s_{1}^{(2)}, s_{1}^{(3)}\right)$ and $\left(s_{2}^{(1)}, s_{2}^{(2)}, s_{2}^{(3)}\right)$ which are also valid. We can restructure any largest feasible set of relay paths into an order-preserving set by repeatedly applying such switching.

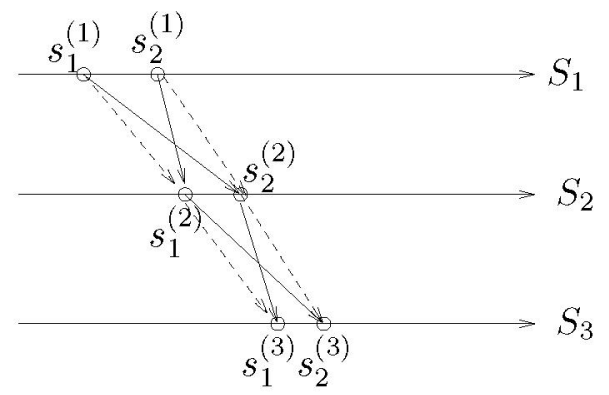

Fig. 1: Dashed lines denote alternative valid relay paths which preserve the order of incoming packets.

Given a sequence of streams $\left(S_{i}\right)_{i=1}^{n+1}$, suppose the attacker wants his traffic to mimic these streams. He needs to find the largest feasible set of relay paths so that he can transmit the maximum number of attacking packets. To this end, we derive an algorithm called "GREEDY-RELAYEMBEDDING" (GRE) for finding the largest feasible set of relay paths. Algorithm GRE is presented in Table 2.

The complexity of GRE is $O\left(n^{3}\left|S_{n+1}\right|\right)$, or more precisely, about $\frac{1}{3}(\lambda \Delta)^{2} n^{3}\left|S_{n+1}\right|$ on the average ${ }^{2}$, where $\lambda$ is the maximum rate of $S_{1}, \ldots, S_{n}$. The set $C_{i, j}$ in GRE is

\footnotetext{
${ }^{2}$ The dominating step is the recursive computation of $C_{i, j}$ 's. There are at most $(n-i+1) \lambda \Delta$ points in $S_{i}$ on the average which are possible to join $C_{i, j}$, and for each of these points, GRE needs no more than
}

Table 2: GREEDY-RELAY-EMBEDDING (GRE).

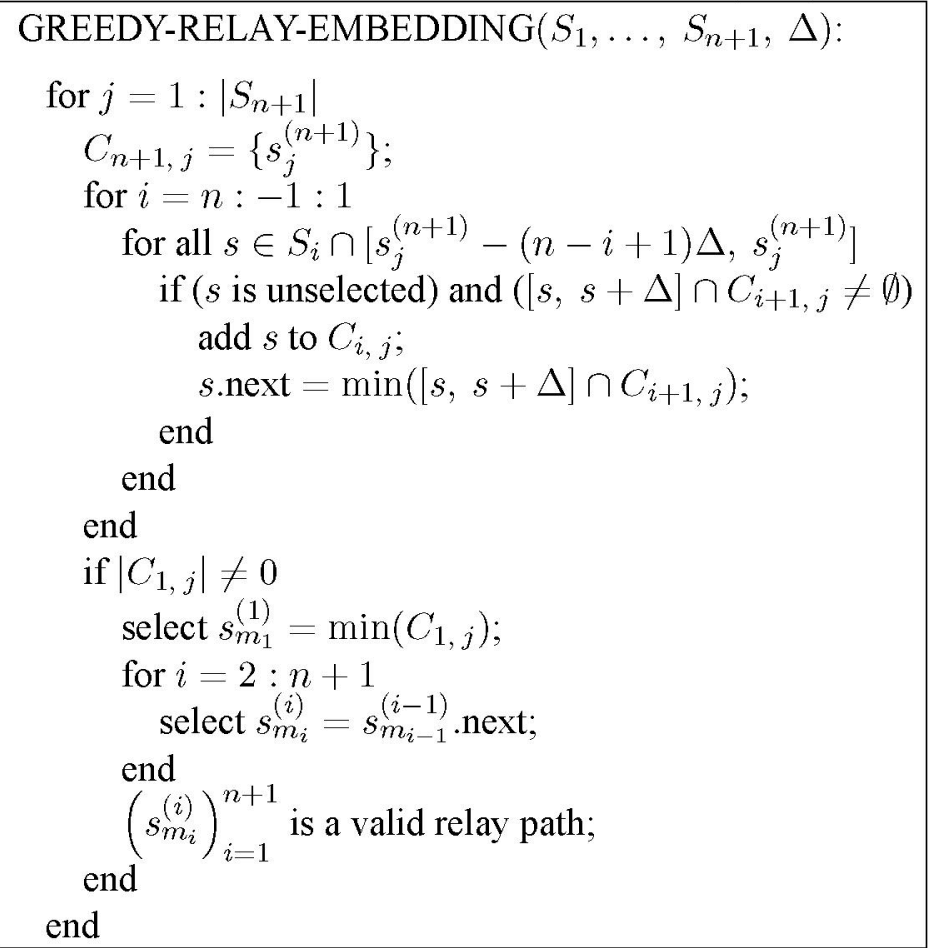

the set of all possible predecessors in $S_{i}$ of the $j$ th point in $S_{n+1}$, i.e.,

$C_{i, j}=\left\{t \in S_{i}: t\right.$ is unselected, and $\exists$ a valid relay path of unselected points from $t$ to $\left.s_{j}^{(n+1)}\right\}$.

Algorithm GRE is based on the idea that among all the valid relay paths for a particular incoming packet, we should choose the earliest one to maximally avoid conflicting with the following incoming packets. For each departing packet from the last host $s_{j}^{(n+1)} \in S_{n+1}$, GRE recursively find the sets $\left\{C_{i, j}\right\}_{i=n}^{1}$ of all its possible predecessors in each of the streams $S_{n}, \ldots, S_{1}$. The construction of $C_{i, j}$ makes sure that every point in it has a valid relay path to $s_{j}^{(n+1)}$, and this path will not conflict with paths that are already selected to relay packets before $s_{j}^{(n+1)}$. If $C_{1, j}$ is not empty, then there must be a valid path from some incoming point in $S_{1}$ to $s_{j}^{(n+1)}$, and GRE selects the earliest of them.

After GRE finds a set of relay paths, the attacker can schedule the transmission of attacking packets accordingly. The unselected points will be the transmission times of chaff

$(n-i) \lambda \Delta$ steps to check the condition $[s, s+\Delta] \cap C_{i+1, j} \neq \emptyset$; GRE needs up to $(n-i)(n-i+1)(\lambda \Delta)^{2}$ steps to compute $C_{i, j}$. The total complexity is then calculated as $\left|S_{n+1}\right| \sum_{i=1}^{n}(n-i)(n-i+1)(\lambda \Delta)^{2} \approx$ $\frac{1}{3}(\lambda \Delta)^{2} n^{3}\left|S_{n+1}\right|$. 
packets. It is easy to see that the set of relay paths found by GRE is feasible. The optimality of GRE is guaranteed by the following proposition.

Proposition 2 Given a realization of point processes $\left(S_{i}\right)_{i=1}^{n+1}$, $G R E$ finds the largest feasible set of relay paths from $S_{1}$ to $S_{n+1}$.

\section{Proof: See Appendix.}

Since GRE is optimal in the sense that it requires the transmission of the minimum number of chaff packets, the performance of GRE gives fundamental limits to the attacker's capability of sending attacking packets. By analyzing GRE, we bound the attacker's ability to send attacking traffic while keeping his traffic completely undetectable to activity-based detectors by adding chaff, as stated in the following theorem.

Theorem 2 Suppose the attacker wants all the streams on the intrusion path to mimic independent Poisson processes with equal rate $\lambda$. Then for an intrusion path of length $n$, the rate of attacking traffic is upper bounded by $\lambda\left(1-e^{-\lambda \Delta}\right)^{n}$.

\section{Proof: See Appendix.}

Remark: Theorem 2 says that if the attacker wants to completely hide the intrusion path, the rate of attacking traffic decays exponentially with the increase in the length of the intrusion path. This result guarantees that the attacker's capability of launching attacks is severely constrained by the number of hops he takes in the chain of stepping stones. To send attacking commands at a sufficiently high rate, The attacker has to either leave some connections on the intrusion path correlated, or reduce the number of stepping stones on the intrusion paths, both of which makes the attacker vulnerable to detection and tracing.

\section{RANDOMIZING PACKET SCHEDULING TO DEFEND AGAINST STEPPING-STONE ATTACKS}

In Section 4, we have established a fundamental limit on the rate of attacking traffic through multiple stepping stones. The result requires that the attacker wants his traffic to mimic Poisson processes. Although we can not control the attacker's decision, as the network designer, we can force the attacker to choose Poisson processes by scheduling other traffic as Poisson. Suppose normal traffic can be modelled as Poisson processes. Then we can install local detectors at the hosts to test whether the interarrival distribution is exponential; all traffic with non-exponential interarrival distributions will be considered abnormal. Next, a global detector can test the dependency among connections to detect stepping-stone traffic. The global detection can be done either in a centralized fashion at a fusion center, or in a distributed fashion by conferencing among local detectors. Using this framework, we show that, at least in principle, scheduling packet transmissions as Poisson processes allows us to restrain the efficiency of stepping-stone attacks.

We note that the key to impeding stepping-stone attacks is to randomize packet transmissions. Randomization gives each traffic flow distinct timing characteristics which can be used to trace the flow. On the other hand, deterministic scheduling does not provide uniqueness in timing, and is therefore vulnerable to encrypted stepping-stone attacks. For example, consider a deterministic scheduling where packets are transmitted after constant interarrival times $D$. If the transmissions are synchronized, then there is no way to distinguish the relay stream from any other stream. Even if the transmissions in streams belonging to independent flows differ by a random time uniformly distributed in $[0, D]$, it is still impossible to distinguish streams with difference within $\Delta$ (assume $\Delta<D$ ). This comparison shows that randomization in transmission times is needed to facilitate the defense against stepping-stone attacks.

The feasibility of the scheduling strategy is also a significant concern. Suppose the ingress traffic of the network can be modelled as Poisson processes. Then it is easy to see that it requires infinite delay and memory to implement a deterministic transmission scheduling, which is infeasible in practice. We point out that Poisson scheduling is not the optimal scheduling to defeat stepping-stone attacks. Take the bounded delay stepping-stone traffic for example; it can be shown that with infinite peak rate (i.e., infinite packets can be transmitted in infinitesimal time), we can design a scheduling strategy to make it almost impossible to embed traffic into independent processes. That is, for any $\epsilon>0$, there exists $\Lambda$, such that there is a scheduling with peak rate bounded by $\Lambda$, and the maximum traffic rate through two independent processes is less than $\epsilon$. Such a bursty scheduling, however, is infeasible in practice because it requires infinite delay and memory to implement as the deterministic scheduling does. Therefore, for large scale networks where the ingress traffic is approximately Poisson, Poisson scheduling is a convenient and effective method to defend against stepping-stone attacks.

\section{CONCLUSION}

In this paper, we show that randomization in packet transmissions facilitates the defense against stepping-stone attacks. The drawback is that such randomization may be undesirable in certain time-sensitive applications such as 
multi-media transmissions. On the other hand, the detection of relayed traffic in time-sensitive applications is an easier problem because the attacker cannot afford to perturb the timing either; see [3].

\section{APPENDIX}

\subsection{Proof of Proposition 2}

By Proposition 1, it suffices to show that GRE finds the largest set of relay paths among all the feasible sets of relay paths that preserve the order of incoming packets.

Let $\mathcal{P}$ be the set of relay paths found by GRE, and $\mathcal{P}^{*}$ a largest feasible set of relay paths that is order-preserving. Suppose $s_{1} \in S_{n+1}$ is the endpoint of a relay path $p_{1}^{*} \in \mathcal{P}^{*}$, as illustrated in Fig. 2, but there is no relay path in $\mathcal{P}$ leading to $s_{1}$. Then in $\mathcal{P}$ there must be relay path(s) having some overlap with $p_{1}^{*}$, and leading to point(s) in $S_{n+1}$ before $s_{1}$; otherwise, GRE would have chosen $p_{1}^{*}$ or some path no later than $p_{1}^{*}$ to lead to $s_{1}$. Let the latest of these points be $s_{2}$, and its path in $\mathcal{P}$ be $p_{1}$. If $s_{2}$ does not correspond to any relay path in $\mathcal{P}^{*}$, we stop tracing; otherwise, let $p_{2}^{*} \in \mathcal{P}^{*}$ lead to $s_{2}$. We know that there have to be relay path(s) in $\mathcal{P}$ partly overlapping with $p_{2}^{*}$; if not, GRE would have chosen a path no later than $p_{2}^{*}$ to lead to $s_{2}$, but this path would not have overlap with $p_{1}^{*}$, which is a contradiction. We continue tracing by alternately choosing the latest path $p_{i}$ in $\mathcal{P}$ which has partial overlap with $p_{i}^{*}$, and then finding a path $p_{i+1}^{*} \in$ $\mathcal{P}^{*}$ with the same endpoint as $p_{i}$ for $i=2,3, \ldots$ The tracing continues until we find a point which has a relay path in $\mathcal{P}$ but not $\mathcal{P}^{*}$, or we reach a relay path $p_{m}$ in $\mathcal{P}$ leading to a point $s_{m+1}$ which is before the endpoint $s_{m}$ of the first relay path $p_{m}^{*}$ in $\mathcal{P}^{*}$.

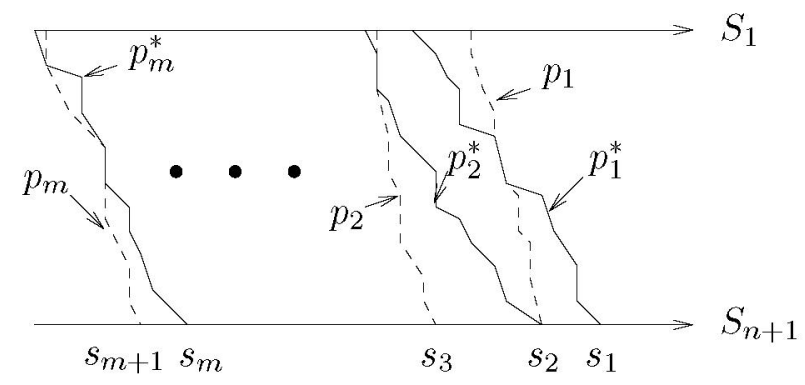

Fig. 2: Every relay path in $\mathcal{P}^{*}$ corresponds to a path in $\mathcal{P}$; solid line: paths in $\mathcal{P}^{*}$; dashed line: paths in $\mathcal{P}$.

Therefore, we see that every relay path in $\mathcal{P}^{*}$ corresponds to a relay path in $\mathcal{P}$. This proves that $\mathcal{P}$ is also a largest feasible set of relay paths.

\subsection{Proof of Theorem 2}

We bound the rate of attacking traffic by obtaining an upper bound on the asymptotic fraction of attacking packets in $S_{1}$. We first show that this fraction is upper bounded by the probability that the first incoming packet can be an attacking packet, and then bounded this probability.

Be Proposition 2, it suffices to bound the fraction of attacking packets scheduled by GRE. For an incoming packet $s_{k}^{(1)}(k \geq 2)$, given a feasible and order-preserving set of relay paths for incoming packets before $s_{k}^{(1)}$ found by GRE, the conditional probability for $s_{k}^{(1)}$ to have a valid relay path is equal to

$$
\operatorname{Pr}\left\{\exists\left(t_{i} \in S_{i}\right)_{i=2}^{n+1}, t_{i} \in\left[\max \left(t_{i-1}, t_{i}^{\prime}\right), t_{i-1}+\Delta\right]\right\},
$$

where $t_{1}=s_{k}^{(1)}$, and $t_{i}^{\prime}$ is the latest point in $S_{i}$ which has been selected by GRE. The condition $t_{i} \geq t_{i}^{\prime}$ represents the order-preserving requirement. We can easily bound this probability from above by

$$
\operatorname{Pr}\left\{\exists\left(t_{i} \in S_{i}\right)_{i=2}^{n+1}, t_{i} \in\left[t_{i-1}, t_{i-1}+\Delta\right]\right\},
$$

which is equal to the probability that $s_{1}^{(1)}$ has a valid relay path.

Next we prove by induction that the probability for $s_{1}^{(1)}$ to have a valid relay path of length $n$ is equal to $\left(1-e^{-\lambda \Delta}\right)^{n}$. Let $t_{1}=s_{1}^{(1)}$. For $n=1$, we have

$$
\operatorname{Pr}\left\{\exists t_{2} \in S_{2}, t_{2} \in\left[t_{1}, t_{1}+\Delta\right]\right\}=1-e^{-\lambda \Delta} .
$$

Assume that the result holds for relay path of length $n-1$ $(n \geq 2)$. Then we have

$$
\begin{aligned}
& \operatorname{Pr}\left\{\exists\left(t_{i} \in S_{i}\right)_{i=2}^{n+1}, t_{i} \in\left[t_{i-1}, t_{i-1}+\Delta\right]\right\} \\
& =\int_{0}^{\Delta} \lambda e^{-\lambda x} \operatorname{Pr}\left\{\exists\left(t_{i} \in S_{i}\right)_{i=2}^{n+1}, t_{i} \in\left[t_{i-1}, t_{i-1}+\Delta\right]\right. \\
& =\int_{0}^{\Delta} \lambda e^{-\lambda x}\left(1-e^{-\lambda \Delta}\right)^{n-1} d x \\
& =\left(1-t_{1}=x\right\} d x
\end{aligned}
$$

where we use the induction assumption in (1).

Combining the facts that $S_{1}$ has rate $\lambda$, and at most (1$\left.e^{-\lambda \Delta}\right)^{n}$ fraction of the packets are attacking packets, we conclude that the rate of attacking traffic is upper bounded by $\lambda\left(1-e^{-\lambda \Delta}\right)^{n}$. 


\section{REFERENCES}

[1] S. Staniford-Chen and L. Heberlein, "Holding intruders accountable on the internet," in Proc. the 1995 IEEE Symposium on Security and Privacy, (Oakland, CA), pp. 39-49, May 1995.

[2] X. Wang, D. Reeves, S. Wu, and J. Yuill, "Sleepy watermark tracing: An active network-based intrusion response framework," in Proc. of the I6th International Information Security Conference, pp. 369-384, 2001.

[3] Y. Zhang and V. Paxson, "Detecting stepping stones," in Proc. the 9th USENIX Security Symposium, pp. 171-184, August 2000.

[4] K. Yoda and H. Etoh, "Finding a connection chain for tracing intruders," in 6th European Symposium on Research in Computer Security, Lecture Notes in Computer Science 1895, (Toulouse, France), October 2000.

[5] X. Wang, D. Reeves, and S. Wu, "Inter-packet delaybased correlation for tracing encrypted connections through stepping stones," in 7th European Symposium on Research in Computer Security, Lecture Notes in Computer Science 2502, pp. 244-263, 2002.

[6] D. Donoho, A. Flesia, U. Shankar, V. Paxson, J. Coit, and S. Staniford, "Multiscale stepping-stone detection: Detecting pairs of jittered interactive streams by exploiting maximum tolerable delay," in 5th International Symposium on Recent Advances in Intrusion Detection, Lecture Notes in Computer Science 2516, 2002.

[7] X. Wang and D. Reeves, "Robust correlation of encrypted attack traffic through stepping stones by manipulation of inter-packet delays," in Proc. of the 2003 ACM Conference on Computer and Communications Security, pp. 20-29, 2003.

[8] A. Blum, D. Song, and S. Venkataraman, "Detection of Interactive Stepping Stones: Algorithms and Confidence Bounds," in Conference of Recent Advance in Intrusion Detection (RAID), (Sophia Antipolis, French Riviera, France), September 2004.

[9] T. He and L. Tong, "A Signal Processing Perspective to Stepping-stone Detection," in Proc. 2006 Conference on Information Sciences and Systems, (Princeton, NJ), March 2006.
[10] P. Peng, P. Ning, D. Reeves, and X. Wang, "Active Timing-Based Correlation of Perturbed Traffic Flows with Chaff Packets," in Proc. 25th IEEE International Conference on Distributed Computing Systems Workshops, (Columbus, OH), pp. 107-113, June 2005.

[11] L. Zhang, A. Persaud, A. Johson, and Y. Guan, "Detection of Stepping Stone Attack under Delay and Chaff Perturbations," in Proc. of the 25th IEEE International Performance Computing and Communications Conference (IPCCC 2006), (Phoenix, AZ), April 2006.

[12] T. He and L. Tong, "Detecting SteppingStone Traffic in Chaff: Fundamental Limits and Robust Algorithms," Tech. Rep. ACSPTR-06-06-01, Cornell University, June 2006. http: / / acsp. ece. cornell . edu/pubR.html.

\footnotetext{
The views and conclusions contained in this document are those of the authors and should not be interpreted as representing the official policies, either expressed or implied, of the Army Research Laboratory or the U. S. Government.
} 